\title{
Fulminant Cerebral Malaria in a Swiss Patient
}

\author{
K. Muehlethaler, E. Scheurer, U. Zollinger, R. Markwalder, X.M. Nguyen
}

\begin{abstract}
Malaria remains the most important parasitic disease worldwide. Falciparum malaria is a medical emergency and requires immediate diagnosis and treatment. Cerebral malaria is a rapidly progressive, potentially fatal complication of Plasmodium falciparum infection. This case, including postmortem observations, histology, and laboratory diagnosis, emphasizes the necessity of appropriate advice regarding malaria prophylaxis before travel to an endemic area. Malaria should always be considered in the differential diagnosis of patients presenting with fever and/or nonspecific flu-like symptoms after traveling to endemic countries.
\end{abstract}

Infection 2005; 33: 33-35

DOI 10.1007/s15010-005-3166-9

\section{Introduction}

Human malaria infection is caused by four species of Plasmodium parasites [1]. Plasmodium falciparum is responsible for at least one million deaths a year [2, 3]. Cerebral malaria is a rapidly progressive, potentially fatal complication of $P$. falciparum infection and a primary cause of sudden death in nonimmunized persons during or following travel in endemic areas. The population at risk consists of tourists, business travelers, and sailors [4]. We present this case as a reminder for colleagues who are only infrequently confronted with such situations.

\section{Case Report}

A previously healthy 34-year-old Caucasian male traveled to Kenya (East Africa), spending 8 days in the coastal area close to Mombassa. Because he booked a last-minute flight 4 days before departure, there was no time to seek pretravel health advice. He departed to an endemic, high-risk area for malaria tropica without obtaining chemoprophylaxis. Two weeks after returning to Switzerland, he complained of an abrupt onset of fever, chills, malaise, and muscle aches. The following day, the patient was treated for influenza by a general practitioner due to flu-like symptoms during the ongoing influenza season in Switzerland. Four days after seeing his doctor, the patient was found dead in his home.

\section{Postmortem Findings (Forensic Autopsy)}

A forensic autopsy revealed a massive congestion and edema of the brain (Figure 1), lungs, and inner organs. The spleen was enlarged with a tense, smooth capsule and a congested parenchyma of brown-black color. Histology showed cerebral grey and white matter with congested capillaries (Figures 2 and 3) containing numerous parasitized erythrocytes (trophozoites). Each cell contained dots of hemozoin pigment.

\section{Laboratory Diagnosis}

Eoletic acid (EDTA) preserved blood was collected postmortem. The percentage parasitemia was about $25 \%$. In both thick and thin Giemsa stained blood smears, numerous trophozoites (merozoites) were found (Figures 4 and 5). P. falciparum antigen (HRP-2, aldolase) was detected by means of an immunochromatographic assay (NOW ${ }^{\circledR}$ Malaria Binax, Binax Inc., Portland, ME, USA).

The differentiation of the trophozoites in the thin Giemsa smear and the $P$. falciparum antigen revealed $P$. falciparum malaria. The observation of microthrombi, consisting of infected erythrocytes, on the cerebral capillary walls established the diagnosis of cerebral malaria. On the basis of histology and macroscopic findings, the patient was considered to have died of fulminant cerebral malaria with multiple organ failure.

\section{Discussion}

This case report shows a fulminant course of complicated malaria tropica with cerebral involvement and multiple organ failure. The case is remarkable because major steps concerning pretravel health advice, clinical diagnosis, and treatment were missed. The patient did not seek pretravel health advice; consequently he did not take any prophy-

\footnotetext{
K. Muehlethaler (corresponding author), X. M. Nguyen Institute for Infectious Diseases, Clinical Microbiology, University of Bern, Friedbuehlstr. 51, CH-3010 Bern, Switzerland; Phone: (+41/31) 632-9979, Fax:-4966, e-mail: konrad.muehlethaler@ifik.unibe.ch E. Scheurer, U. Zollinger Institute of Forensic Medicine, University of Bern, Bern, Switzerland R. Markwalder

Institute for Pathology, University of Bern, Bern, Switzerland
} 


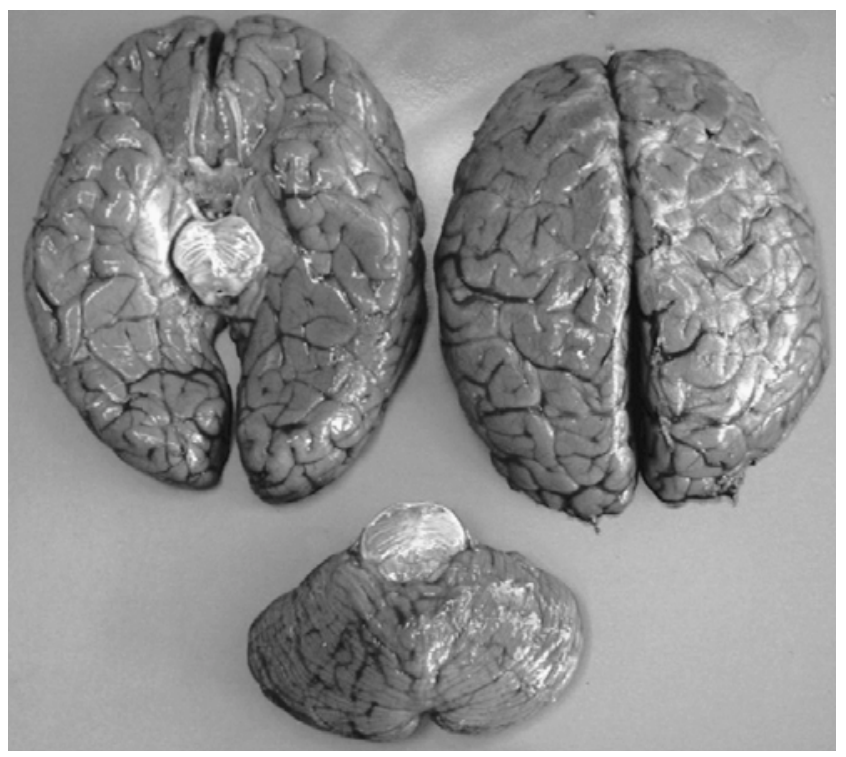

Figure 1. Brain: massive congestion and edema.

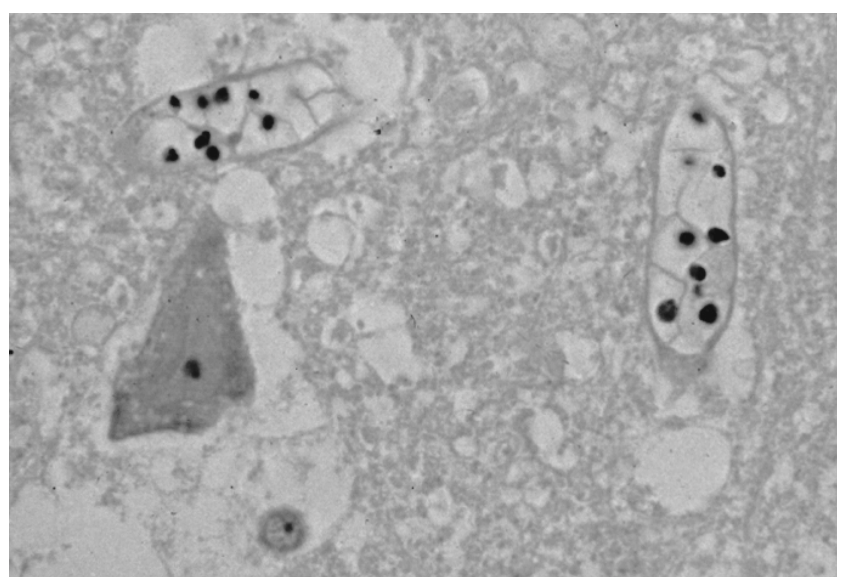

Figure 2. Brain: neuron and congested vessels with trophozoites in red blood cells (Giemsa, oil $400 \times$ ).

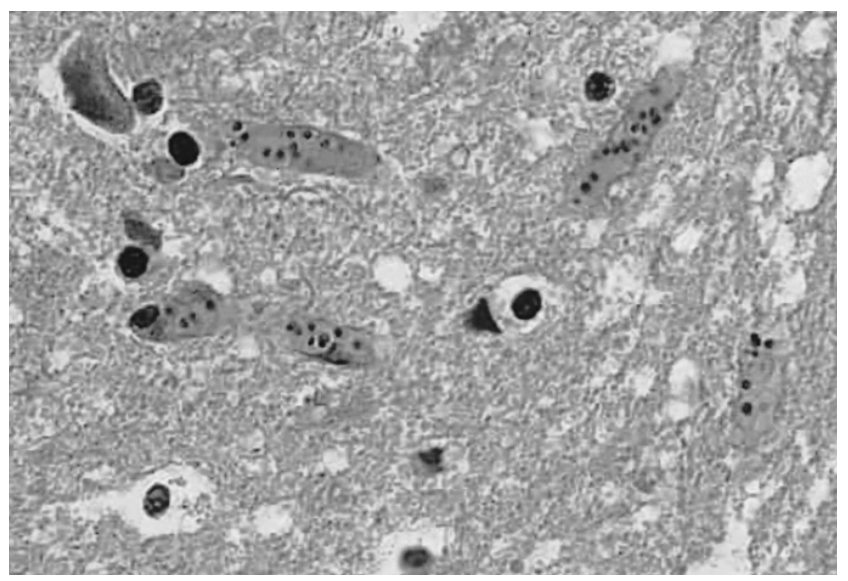

Figure 3. Brain: capillaries plugged with trophozoites in red blood cells $(\mathrm{HE}, 250 \times)$. laxis. After returning from an endemic area, malaria was not considered in the differential diagnosis although the man presented with fever.

Due to a worldwide increase in travel to tropical countries, a similar increase in travel-related morbidity could be expected. There is, therefore, a need for effective, accessible, and appropriate pretravel health advice [5]. In addition to vaccination against other diseases, the patient must be informed about personal protection measures (repellents, mosquito nets, clothes, etc.) and chemoprophylaxis or standby therapy for malaria, according to the destination. Because of the constantly changing situation with regard to drug-resistant malaria, it is mandatory to keep up to date regarding current recommendations [6]. Alternatively, the patient may be referred to a tropical medicine or travel specialist.

The classic clinical presentation of malaria consists of fever accompanied by various other symptoms such as headache, malaise, nausea, muscular pain, and occasionally diarrhea. This clinical picture can easily be mistaken

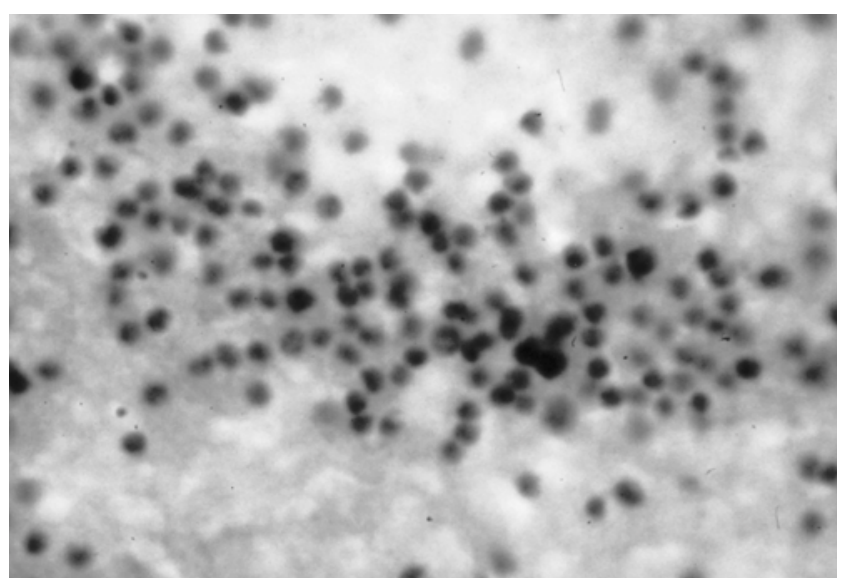

Figure 4. Thick blood smear (Giemsa, $1000 \times$ oil).

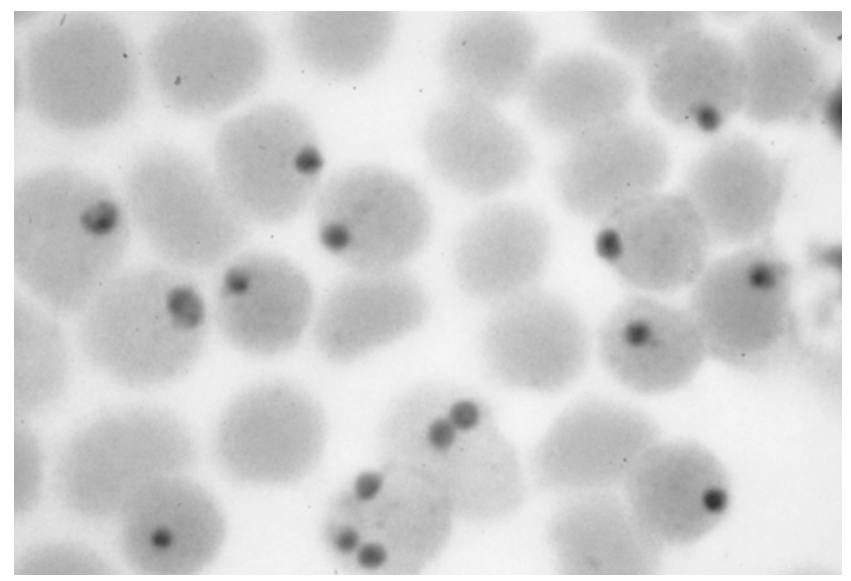

Figure 5. Thin blood smear (Giemsa, $1000 \times$ oil). 
for influenza, especially during winter when influenza is highly prevalent [7]. Therefore, it is important to recognize cases promptly and to identify the pathogens to species level. Falciparum malaria in a nonimmune patient is a medical emergency and requires immediate diagnosis and treatment in a hospital setting. The case fatality rate of strictly defined cerebral malaria in endemic areas remains of the order of $20 \%$ in adults and $15 \%$ in children [2]. Until proven otherwise, malaria must be suspected in all febrile people returning from malaria-endemic areas; therefore a detailed travel history is mandatory. Delays in diagnosis and treatment increase the risk of complications and mortality $[2,8,9]$.

\section{Acknowledgment}

We thank W. John Looney, FIBMS, for reviewing this article.

\section{References}

1. Turner G: Cerebral malaria. Brain Pathol 1997; 7: 569-582.

2. Warrell DA: Cerebral malaria: clinical features, pathophysiology and treatment. Ann Trop Med Parasitol 1997; 91: 875-884.
3. Murray CJ, Lopez AD: Mortality by cause for eight regions of the world: Global Burden of Disease Study. Lancet 1997; 349: 1269-1276.

4. Yapo Ette H, Koffi K, Botti K, Jouvet A, Effi AB, Honde M: Sudden death caused by parasites: postmortem cerebral malaria discoveries in the African endemic zone. Am J Forensic Med Pathol 2002; 23: 202-207.

5. Hoveyda N, Behrens R: More travel advice and fewer vaccinations are needed. BMJ 2003; 326: 52.

6. Humar A, Sharma S, Zoutman D, Kain KC: Fatal falciparum malaria in Canadian travellers. Can Med Assoc J 1997; 156: 1165-1167.

7. Albert S, Schroter A, Bratzke H, Brade V: Postmortem diagnosis of tropical malaria. Dtsch Med Wochenschr 1995; 120: 18-22.

8. Lamparter S, Schoner K, Moll R, Mennel HD, Maisch B: Fulminant course of falciparum malaria. Dtsch Med Wochenschr 2001; 126: 76-78.

9. Winters RA, Murray HW: Malaria - the mime revisited: fifteen more years of experience at a New York City teaching hospital. Am J Med 1992; 93: 243-246. 